УДК 510.643

\title{
Unification in Linear Modal Logic on Non-transitive Time with the Universal Modality
}

\author{
Stepan I. Bashmakov* \\ Institute of Mathematics and Computer Science, \\ Siberian Federal University, \\ Svobodny 79, Krasnoyarsk, 660041
}

Russia

Received 05.08.2017, received in revised form 10.09.2017, accepted 20.10.2017

\begin{abstract}
We investigate the question of unification in the linear modal logic on non-transitive time with the universal modality. The semantic construction of logic on linear non-transitive Kripke frames is proposed, effective definability and projectivity of the unifiable formulas are proved. An algorithm for construction the most general unifier is found.

Keywords: temporal modal logic, non-transitive Kripke frame, unification, ground unifier, projective formulas.

DOI: 10.17516/1997-1397-2018-11-1-3-9.
\end{abstract}

\section{Introduction}

Modal logics play an important role in the design of systems that provide components of reasoning about the knowledge and time. Temporal logics actively develops the fields of mathematical logic, philosophy, computer science and artificial intelligence. The first study of temporal logics as modal systems was proposed by A. Prior [1], for the next half-century this area has become a complex technical discipline [2].

The idea of non-transitive time, in the aspect of knowledge, proceeds from the observation that the transfer of knowledge from the past to the future may not always be successfully performed: the available information in the past may not be available in the present. A detailed consideration of different points of view on non-transitive time and its expression by means of logical systems is considered in [3].

At the stage of its formation, the unification problem consisted in answering the question: is it possible to transform two terms into syntactically equivalent ones by changing variables to other terms. In the field of nonstandard logics this problem is equivalent to (and more often considered in the form of) possibility of a formula to become a theorem after replacing variables, preserving the values of the coefficient-parameters [4].

V.V. Rybakov solved this problem for modal $\mathcal{S} 4, \mathcal{G} r z$ and intuitionistic logics, [5], in [6] he proposed an approach to the definition of all non-unifiable formulas for the extensions of $S 4$ and $(K 4+[\square \perp \equiv \perp])$. Using this technique, the criteria of non-unifiability in linear transitive temporal logics of knowledge with multi-modal relations were found: over the $\mathbb{N}(\mathcal{L T} \mathcal{K},[7])$ and over the $\mathbb{Z}$ with alternative relations $(\mathcal{L} \mathcal{F} \mathcal{P} \mathcal{K},[8])$.

\footnotetext{
*krauder@mail.ru

(C) Siberian Federal University. All rights reserved
} 
To study the unification S. Ghilardi proposed a new approach based on the projective formulas [9], which allowed to algorithmize the construction of a finite complete sets of unifiers for the series of logics, [10,11]. Based on this approach, W. Dzik and P. Wojtylak established a projective unification in the extensions of the logic $\mathcal{S} 4.3$ [12]. In [13-16] it was found that a solution of the admissibility problem follows from the existence of computable complete sets of unifiers, which significantly increased the importance of the approach to unification through the projective formulas. In [17] V. V. Rybakov explored the modification of linear temporal logic $\mathcal{L} \mathcal{T}$ with the operator $U n t i l$, for which the projective unification was established. From the projectivity of unification follows the existence of the most general unifier (mgu), but not vice versa. For example, in [18] the existence of mgu for each unified formula in $\mathcal{L} \mathcal{T} \mathcal{L}$ with the operators $N$ ext and $U$ til is proved and counterexample is constructed: an unified, but not a projective formula. In [19] the projective unification is proved for $\mathcal{L} \mathcal{F} \mathcal{P} \mathcal{K}, \mathcal{L} \mathcal{F} \mathcal{P} \mathcal{K}_{U_{-}}^{U_{+}}, \mathcal{L} \mathcal{F} \mathcal{P} \mathcal{K}_{U_{-}, P}^{U_{+}, N}$.

The unification problem is reducible to the admissibility problem: the formula $\varphi$ is unifiable in the $\operatorname{logic} \mathcal{L}$ if the inference rule $\varphi / \perp$ is not admissible in $\mathcal{L}$. In some cases, when logic has a finitary type of unification, the admissibility problem is also reducible to the problem of unification $[20,21]$.

The approach based on the construction of a ground unifier (i.e., obtained by the substitution of constants) demonstrates wide applicability: both in a way of proving the unifiability of an arbitrary formula, and in constructing projective unifiers [17, 19,22]. The idea of constructing a projective unifier using a ground one, however, is not universal and all-applicable: in [10] it was shown that not for every formula in $\mathcal{I}$ nt a ground unifier gives a construction of a projective unifier, in [12] it was proved that for $\mathcal{S} 4.3$ the projective unifier can't be simply described on the basis of the ground one. Despite this, the use of ground unifiers in solving unification problems is stay appropriate even when logic has nullary (worst) type of unification and mgu for some formulas do not exist: the construction of the ground unifier remains possible.

Simultaneously with intensive studies of unification in transitive logics, analogous questions remain extremely poorly studied for non-transitive cases, where they appear to carry much greater complexity, and many methods and even definitions turns out to be inapplicable or require considerable modification. However, it would be unfair ignore the existence of works for logics with non-standard relations. For example, E. Jerabek proved the nullary type of unification in minimal normal logic $\mathcal{K}[23]$, and W. Dzik - the best - unitary type for $\mathcal{S} 5$ and its extensions [22]. F. Wolter and M. Zakharyaschev [24] proved the unsolvability of unification over the $\mathcal{K}$ with additional universal modality.

In this paper, we investigate linear modal logic based on non-transitive time with a universal modality. It is proved that unifiability of any formula in this logic can be effectively detemined and a ground unifier can be found, if one exists. The projective unification is established, which guarantees its unitary type [9] and (almost) structurally completeness [25] in this logic.

\section{Definitions and semantics}

We give some definitions and formulations, and also semantic construction of linear bimodal logic of non-transitive time with universal modality (in our notation $\mathcal{U L \mathcal { T }} \mathcal{L}$ ).

The alphabet of the language $L^{\mathcal{U L I T} \mathcal{L}}$ includes a countable set of propositional variables $P=\left\{p_{1}, \ldots, p_{n}, \ldots\right\}$, brackets $($,$) , standard Boolean operations and two modal operators: non-$ transitive $\diamond$ and universal $\square_{U}$ modalities.

Kripke frame (or scale) $F$ is a pair $\langle W, R\rangle$, where $W$ is a nonempty set of elements, and $R$ is 
a binary relation on $W$. If for $a, b \in W$ is fulfilled $a R b$, then say $a$ «sees» $b$. A frame $F$ is said to be reflexive and transitive if its binary relation $R$ is such kind. Let $\left\{p_{1}, \ldots, p_{n}\right\}$ be the set of propositional variables. Valuation $V$ on the frame $F$ is a mapping associating with each variable $p_{i}$ subset $V\left(p_{i}\right) \subseteq W$. Kripke model $M$ (or shortly model) is a triple $\langle W, R, V\rangle$, where $\langle W, R\rangle$ is a frame, and $V$ is a valuation of propositional variables from the set $\operatorname{Dom}(V)=\left\{p_{1}, \ldots, p_{n}\right\}$ called sign domain $V$. Let the model $M=\langle F, V\rangle$ be given. Then $\forall w \in F$ :

a. $\langle F, w\rangle \Vdash_{V} p \Leftrightarrow w \in V(p)$;

b. $\langle F, w\rangle \Vdash_{V} \varphi \vee \psi \Leftrightarrow\left[\left(\langle F, w\rangle \Vdash_{V} \varphi\right) \vee\left(\langle F, w\rangle \Vdash_{V} \psi\right)\right]$;

c. $\langle F, w\rangle \Vdash_{V} \varphi \wedge \psi \Leftrightarrow\left[\left(\langle F, w\rangle \Vdash_{V} \varphi\right) \wedge\left(\langle F, w\rangle \Vdash_{V} \psi\right)\right]$;

d. $\langle F, w\rangle \Vdash_{V} \neg \varphi \Leftrightarrow\left[\neg\left(\langle F, w\rangle \Vdash_{V} \varphi\right)\right]$;

e. $\langle F, w\rangle \Vdash_{V} \diamond \varphi \Leftrightarrow\left[\exists v \in F:(w R v) \Rightarrow\left(\langle F, v\rangle \Vdash_{V} \varphi\right)\right]$;

f. $\langle F, w\rangle \Vdash_{V} \square \varphi \Leftrightarrow\left[\forall v \in F:(w R v) \Rightarrow\left(\langle F, v\rangle \Vdash_{V} \varphi\right)\right]$.

For the logic $\mathcal{L}$ a frame $F$ is called a $\mathcal{L}$-frame or a frame that is adequate to the logic $\mathcal{L}$, if for any formula $\alpha \in \mathcal{L}$ for any valuation $V$ we have $F \Vdash_{V} \mathcal{L}$. The logic $\mathcal{L}$ defined by the frame $F$ will be written as $\mathcal{L}(F)$.

In this paper we consider the Kripke frame $F=\left\langle\mathbb{N}, N e x t_{i n f}\right\rangle$, where $\mathbb{N}$ is the set of integers, and $N e x t_{\text {inf }}$ is the binary relation «next natural number»: $\forall a, b \in \mathbb{N}: a N \operatorname{ext}_{\text {inf }} b \Leftrightarrow b=a+1$. The model on the infinite frame $F=\left\langle\mathbb{N}, N e x t_{\text {inf }}\right\rangle$ will be denoted as $M=\langle F, V\rangle$.

In accordance with the definition, the frame $F$ is linear non-transitive with irreflexive points, therefore the truth values of the modality $\square$ on any such model $M$ coincides with $\diamond$.

In addition to the non-transitive modality $\diamond$, the language of logic $\mathcal{U L \mathcal { L }} \mathcal{L}$ contains the modal operator $\square_{U}$, the truth values of formulas containing $\square_{U}$ on $M=\langle F, V\rangle$ is given as follows:

$$
\forall x \in F,\langle F, x\rangle \Vdash_{V} \square_{U} \varphi \leftrightarrow\left[\forall y \in F,\langle F, y\rangle \Vdash_{V} \varphi\right]
$$

The modal operator $\diamond_{U}$ is expressed in terms of the pairwise $\square_{U}$ by the ordinary way: $\diamond_{U} \varphi:=\neg \square_{U} \neg \varphi$.

In other words, $\square_{U} \varphi$ means that the formula $\varphi$ always and everywhere valid. In this case, $\square_{U}$ is called a universal modality, and logic $\mathcal{U L \mathcal { L }} \mathcal{T} \mathcal{L}$ containing $\square_{U}$ is called the linear bimodal logic based on non-transitive time with universal modality.

Definition 1. Logic $\mathcal{U L I T} \mathcal{L}$ is the set of all formulas of the language $L^{\mathcal{U} \mathcal{L} \mathcal{T} \mathcal{L}}$ valid on the frame $F$ :

$$
\mathcal{U L \mathcal { T }} \mathcal{L}:=\left\{A \in \operatorname{Fma}\left(L^{\mathcal{U} \mathcal{L} \mathcal{I} \mathcal{L}}\right) \mid F \in \mathcal{U} \mathcal{L} \mathcal{I} \mathcal{T} \mathcal{L}\left(F \Vdash_{V} A\right)\right\}
$$

\section{Unification}

The length $l(\alpha)$ of the formula $\alpha$ is defined as follows: $l(p)=0$, where $p$ is a proposition variable; $l(\alpha \circ \beta)=l(\alpha)+l(\beta)+1$, where $\circ \in\{\vee, \wedge\} ; l(\bigcirc \alpha)=l(\alpha)+1$, where $\circ \in\left\{\neg, \diamond, \square_{U}\right\}$.

Before proceeding to the main results, we prove an auxiliary, almost obvious, fact.

Proposition 1. For all $c_{1}, \ldots, c_{r} \in\{\top, \perp\}$ and any formula $\delta\left(p_{1}, \ldots, p_{r}\right)$ there is $c \in\{\top, \perp\}$, s.t. $\forall x \in F,\langle F, x\rangle \Vdash \delta\left(c_{1}, \ldots, c_{r}\right) \equiv c$.

Proof. We carry out the proof by induction on the length of the formula $\delta$. Let $\delta=p$, then as a result of the substitution we get $\delta=\top$, so $V(\top)=F$, or $\delta=\perp$, which means $V(\perp)=\varnothing$.

If $\delta=c_{1} \vee c_{2}$, where $c_{1}, c_{2} \in\{\top, \perp\}$, then $\delta=\max \left(c_{1}, c_{2}\right)$, if $\delta=c_{1} \wedge c_{2}$, then $\delta=\min \left(c_{1}, c_{2}\right)$ and, by the inductive hypothesis, $V(\delta)=F$ or $V(\delta)=\varnothing$. 
If $\delta=\neg c_{1}$, where $c_{1} \in\{\top, \perp\}$, then $\delta=\top$, if $c_{1}=\perp$, or $\delta=\perp$, if $c_{1}=\top$ and, again accordingly to the inductive hypothesis, $V(\delta)=F$ or $V(\delta)=\varnothing$.

Let $\delta=\bigcirc c_{1}$, where $\bigcirc=\left\{\diamond, \square_{U}\right\}$ and $c_{1} \in\{\top, \perp\}$. If $c_{1}=\perp$ then, because of $V(\perp)=\varnothing$, we get $V(\bigcirc \perp)=\varnothing$. If $c_{1}=\top$ then, because of $V(\top)=F$, we also get $V(\bigcirc \top)=F$.

Definition 2. A formula $\alpha\left(p_{1}, \ldots, p_{s}\right)$ is said to be unifiable in a logic $\mathcal{L}$ iff exists a substitution $\sigma: p_{i} \mapsto \sigma_{i}$ for each $p_{i}$, s.t. $\alpha\left(\sigma_{1}, \ldots, \sigma_{s}\right) \in \mathcal{L}$. In this case, this substitution $\sigma$ is called a unifier of the formula $\alpha$.

A ground unifier is a unifier obtained by the substitution constants $\{\top, \perp\}$ in place of the variables of the formula.

Earlier, in [26] we proved the criterion of non-unifiability for arbitrary $\mathcal{L}$ with expressible universal modality:

Theorem 1. A formula $A$ is non-unifiable in $\mathcal{L} \Leftrightarrow \square_{U} A \rightarrow\left[\bigvee_{p \in \operatorname{Var}(A)} \diamond_{U} p \wedge \diamond_{U} \neg p\right] \in \mathcal{L}$.

Definition 3. A unifier $\sigma$ of the formula $\alpha\left(p_{1}, \ldots, p_{s}\right)$ is called more general than another $\sigma^{1}$ in $\mathcal{L}$, if there exists a substitution $\sigma^{2}$, s.t. for any variable $p_{i}: \sigma^{1}\left(p_{i}\right) \equiv \sigma^{2}\left(\sigma\left(p_{i}\right)\right) \in \mathcal{L}$.

$A$ unifier $\sigma$ of the formula $\alpha\left(p_{1}, \ldots, p_{s}\right)$ is called a most general unifier (shortly mgu), if for any other $\sigma^{i}$ unifier $\sigma$ is more general than $\sigma^{i}$.

A most general unifier can be interpreted as the best solution to the unification problem. Logic has a unitary type of unification, if for any unified formula there is a mgu; finitary (infinitary) type if there is a finite (respectively infinite) number of the best solutions (in this case, all of them are called a maximal unifiers). The worst type of unification is nullary type: some of the unifiable formulas do not have maximal unifiers, [23].

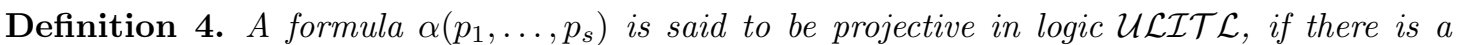
unifier $\tau$ (which called projective unifier) for a formula $\alpha$, s.t. $\square_{U} \alpha \rightarrow\left[p_{i} \equiv \tau\left(p_{i}\right)\right] \in \mathcal{U L \mathcal { L } \mathcal { L }}$ for any variable $p_{i}$ of the formula $\alpha$.

For an arbitrary formula in the $\operatorname{logic} \mathcal{U L \mathcal { T }} \mathcal{L}$ it is possible to establish its unifiability using only ground unifiers:

Theorem 2. Unifiability of an arbitrary formula $\varphi\left(p_{1}, \ldots, p_{s}\right)$ in $\mathcal{U L \mathcal { L }} \mathcal{L}$ can be effectively established using the substitution $\sigma(\varphi)$ of the following form: $\forall p_{i} \in \operatorname{Var}(\varphi) \sigma\left(p_{i}\right) \in\{\top, \perp\}$.

Proof. Lets show that to check unifiability of any given formula $\varphi$ it is enough to establish only the existence of the ground unifier $g u:=\{\top, \perp\}$, obtained by substituting variables for constants.

Let a formula $\varphi\left(p_{1}, \ldots, p_{s}\right)$ be unified in $\mathcal{U} \mathcal{L} \mathcal{I} \mathcal{T} \mathcal{L}$ and the set $\delta_{1}\left(q_{1}, \ldots, q_{r}\right), \ldots, \delta_{s}\left(q_{1}, \ldots, q_{r}\right)$ is its unifier. Then

$$
\delta(\varphi):=\varphi\left(\delta_{1}\left(q_{1}, \ldots, q_{r}\right), \ldots, \delta_{s}\left(q_{1}, \ldots, q_{r}\right)\right) \in \mathcal{U} \mathcal{L} \mathcal{I} \mathcal{T} \mathcal{L}
$$

We replace the variables $q_{1}, \ldots, q_{r}$ by the constants $c_{i} \in\{\top, \perp\}(i \in[1, r])$ in an arbitrary way. Because of we are dealing with a valid formula in logic, as a result of substitution we again obtain valid formula:

$$
\varphi\left(\delta_{1}\left(c_{1}, \ldots, c_{r}\right), \ldots, \delta_{s}\left(c_{1}, \ldots, c_{r}\right)\right) \in \mathcal{U} \mathcal{L} \mathcal{I} \mathcal{T} \mathcal{L}
$$

Let us denote $g u\left(p_{i}\right):=\delta_{i}\left(c_{1}, \ldots, c_{r}\right)$, then

$$
\varphi\left(g u\left(p_{1}\right), \ldots, g u\left(p_{s}\right)\right) \in \mathcal{U} \mathcal{L} \mathcal{I} \mathcal{T},
$$


where each of $g u\left(p_{i}\right) \in\{\top, \perp\}$ is a constant. Therefore, $g u(\varphi)$ is a ground unifier, which existence for an arbitrary formula can be verified in $\mathcal{U L I T} \mathcal{L}$ as follows.

Due to the fact that $g u\left(p_{1}\right), \ldots, g u\left(p_{s}\right)$ in nothing more than a set of constants for which $\varphi$ is true, for an arbitrary (not necessarily unified) formula $\psi\left(p_{1}, \ldots, p_{s}\right)$ it suffices to go through no more than $2^{s}$ substitution options of $\{\top, \perp\}$ instead of variables. If among them there is such that $\psi\left(g u\left(p_{1}\right), \ldots, g u\left(p_{s}\right)\right) \equiv \mathcal{U L \mathcal { I T } \mathcal { L }} \top$, it will mean that formula $\psi$ is unifiable in $\mathcal{U} \mathcal{L} \mathcal{I} \mathcal{T} \mathcal{L}$ and $g u(\psi) \in$ $\mathcal{U} \mathcal{L} \mathcal{I} \mathcal{L}$ is its ground unifier. Otherwise, if for all $2^{s}$ substitution options $g u\left(p_{1}\right), \ldots, g u\left(p_{s}\right)$,

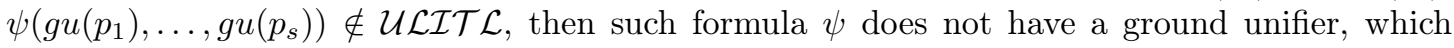
means that it is non-unifiable in $\mathcal{U} \mathcal{L} \mathcal{I} \mathcal{T} \mathcal{L}$.

Now we are ready to prove the main result of the article.

Theorem 3. Any unifiable in $\mathcal{U} \mathcal{L} \mathcal{I} \mathcal{L}$ formula is projective.

Proof. Assuming that $\varphi\left(p_{1}, \ldots, p_{s}\right)$ is unifiable in $\mathcal{U L I T} \mathcal{L}$ formula. For any variable $p_{i} \in \operatorname{Var}(\varphi)$ we set the following substitution $\sigma\left(p_{i}\right)$ :

$$
\sigma\left(p_{i}\right):=\left(\square_{U} \varphi \wedge p_{i}\right) \vee\left(\neg \square_{U} \varphi \wedge g u\left(p_{i}\right)\right),
$$

where $g u\left(p_{1}\right), \ldots, g u\left(p_{s}\right)$ is a ground unifier of the formula $\varphi\left(p_{1}, \ldots, p_{s}\right)$, obtained by the algorithm from the previous theorem.

Take any infinite model $M:=\langle F, V\rangle$ with the arbitrary valuation $V$. If $\sigma$ is a unifier for $\varphi$,

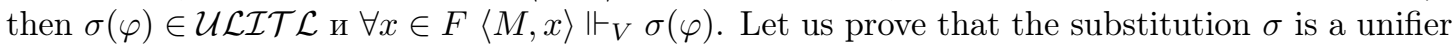

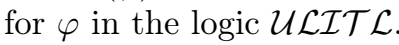

1. If $\forall x \in F:\langle M, x\rangle \Vdash_{V} \varphi$, then $\langle M, x\rangle \Vdash_{V} \square_{U} \varphi$ and hence the second disjunctive term is refuted at $x$. If $\langle M, x\rangle \Vdash_{V} p_{i}$, then $\langle M, x\rangle \Vdash_{V} \square_{U} \varphi \wedge p_{i}$, hence $\langle M, x\rangle \Vdash_{V} \sigma\left(p_{i}\right)$. If $\langle M, x\rangle \Vdash_{V} \neg p_{i}$, then $\langle M, x\rangle \nVdash_{V} \square_{U} \varphi \wedge p_{i}$ and therefore $\langle M, x\rangle \Vdash_{V} \neg \sigma\left(p_{i}\right)$. Since the truth value of the variable $p_{i}$ here depends on the given valuation $V$ at which $\varphi$ is a true on the model $M$, consequently, the truth value of $\varphi\left(p_{1}, \ldots, p_{s}\right)$ at the point $x$ w.r.t. $V$ coincides with the value of $\varphi\left(\sigma\left(p_{1}\right), \ldots, \sigma\left(p_{s}\right)\right)$ at the same point w.r.t. $V$ and hence in this case $\langle M, x\rangle \Vdash_{V} \sigma(\varphi)$.

2. If $\exists x \in F:\langle M, x\rangle \Vdash_{V} \neg \varphi$, then $\langle M, x\rangle \nVdash_{V} \square_{U} \varphi$, which is possible for the second disjunctive term, but the first one is immediately disproved at $x$. Then the truth values of all $\sigma\left(p_{i}\right)$ at $x$ coincide with $g u\left(p_{i}\right)$, and because $\langle M, x\rangle \Vdash_{V} g u(\varphi)$ (by virtue of the selection of the ground unifier $g u(\varphi) \in \mathcal{U L I} \mathcal{L} \mathcal{L})$, again $\langle M, x\rangle \Vdash_{V} \sigma(\varphi)$. Hence, $\sigma(\varphi) \in \mathcal{U} \mathcal{L} \mathcal{I} \mathcal{T} \mathcal{L}$ for the unifiable in $\mathcal{U L I T} \mathcal{L}$ formula $\varphi$.

Lets prove that $\sigma(\varphi)$ is a projective unifier. If we substitute $\sigma\left(p_{i}\right)$ into the definition of the projective formula, we obtain the following: $\forall p_{i} \in \operatorname{Var}(\varphi)$

$$
\square_{U} \varphi \rightarrow\left(p_{i} \leftrightarrow\left[\left(\square_{U} \varphi \wedge p_{i}\right) \vee\left(\neg \square_{U} \varphi \wedge g u\left(p_{i}\right)\right)\right]\right) \in \mathcal{U} \mathcal{L} \mathcal{I} \mathcal{T} \mathcal{L},
$$

if $\sigma$ is a projective unifier for $\varphi$. Assume the converse: let $\sigma$ be a not projective substitution. Then for some model $M \exists x \in M$

$$
\langle M, x\rangle \Vdash_{V} \square_{U} \varphi,
$$

but

$$
\langle M, x\rangle \nVdash_{V} p_{i} \leftrightarrow\left[\left(\square_{U} \varphi \wedge p_{i}\right) \vee\left(\neg \square_{U} \varphi \wedge g u\left(p_{i}\right)\right)\right] .
$$

In this case

$$
\langle M, x\rangle \nVdash_{V} p_{i} \rightarrow\left[\left(\square_{U} \varphi \wedge p_{i}\right) \vee\left(\neg \square_{U} \varphi \wedge g u\left(p_{i}\right)\right)\right],
$$

or

$$
\langle M, x\rangle \nVdash_{V}\left[\left(\square_{U} \varphi \wedge p_{i}\right) \vee\left(\neg \square_{U} \varphi \wedge g u\left(p_{i}\right)\right)\right] \rightarrow p_{i} .
$$

If (3), then $\langle M, x\rangle \Vdash_{V} p_{i}$, but in this instance $\langle M, x\rangle \Vdash_{V} \square_{U} \varphi \wedge p_{i}$, by virtue of (1) and $p_{i}$ at $x$, and therefore $\langle M, x\rangle \Vdash_{V} p_{i} \rightarrow\left[\left(\square_{U} \varphi \wedge p_{i}\right) \vee\left(\neg \square_{U} \varphi \wedge g u\left(p_{i}\right)\right)\right]$. 
If (4), consequently $\langle M, x\rangle \Vdash_{V}\left[\left(\square_{U} \varphi \wedge p_{i}\right) \vee\left(\neg \square_{U} \varphi \wedge g u\left(p_{i}\right)\right)\right]$, but it is possible only with $\langle M, x\rangle \Vdash_{V} p_{i}$, because $\langle M, x\rangle \Vdash_{V} \square_{U} \varphi$ following from (1), hence in the disjunction of $\sigma\left(p_{i}\right)$ only first term can be fulfilled. Therefore the conclusion (4) is true and $\langle M, x\rangle \Vdash_{V}\left[\left(\square_{U} \varphi \wedge p_{i}\right) \vee\right.$

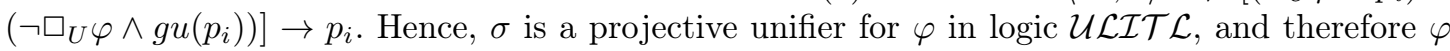
is a projective formula.

By the theorem above, for any $\varphi$ unifiable in $\mathcal{U L I} \mathcal{L} \mathcal{L}$ the substitution $\sigma$ is a projective unifier, and hence the most general one [9]. Besides, the existence of mgu for every unified formula implies the finiteness of all complete sets of unifiers in the logic, and all of them can be obtained from the given projective substitution $\sigma$, and the logic $\mathcal{U L \mathcal { I }} \mathcal{L}$ has a unitary type of unification [9].

A remarkable consequence of the projective unification in the logic $\mathcal{U L \mathcal { T }} \mathcal{L}$ is also its almost structurally completeness [25]: each admissible rule in $\mathcal{U L} \mathcal{I} \mathcal{T} \mathcal{L}$ is derivable.

\section{References}

[1] A.Prior, Time and Modality, Oxford University Press, 1957.

[2] D.M.Gabbay, I.M.Hodkinson, M.A.Reynolds, Temporal Logic: Mathematical Foundations and Computational Aspects, Clarendon Press, Oxford, vol. 1, 1994.

[3] V.Rybakov, Intransitive Linear Temporal Logic, Knowledge from Past, Decidability, Admissible Rules, CoRR, 2015, http://arxiv.org/abs/1503.08761.

[4] S.P.Odintsov, V.V.Rybakov, Unification Problem in Nelson's Logic N4, Sib. Electronic Math. Reports, 11(2014), 434-443.

[5] V.V.Rybakov, Admissible Logical Inference Rules. Series: Studies in Logic and the Foundations of Mathematics, Elsevier Sci. Publ., North-Holland, vol. 136, 1997.

[6] V.V.Rybakov, M.Terziler, C. Gencer, An essay on unification and inference rules for modal logics, Bulletin of the Section of Logic, 28(1999), no. 3, 145-157.

[7] S.I.Bashmakov, Unification and inference rules in the multi-modal logic of knowledge and linear time LTK, Journal of SibFU. Mathematics and Physics, 9(2016), no. 2, 149-157.

[8] S.I.Bashmakov, A.V. Kosheleva, V. Rybakov, Non-unifiability in linear temporal logic of knowledge with multi-agent relations, Sib. Electronic Math. Reports, 13(2016), 923-929.

[9] S.Ghilardi, Unification Through Projectivity, J. Logic Comput., 7(1997), no. 6, 733-752.

[10] S.Ghilardi, Unification in Intuitionistic logic, J. Symbolic Logic, 64(1999), no. 2, 859-880.

[11] S.Ghilardi, L.Sacchetti, Filtering Unification and Most General Unifiers in Modal Logic, J. Symbolic Logic, 69(2004), no. 3, 879-906.

[12] W.Dzik, P.Wojtylak, Projective unification in modal logic, Logic J. IGPL, 20(2012), no. 1, $121-153$.

[13] E.Jerábek, Admissible rules of modal logics, J. Logic Comput., 15(2005), 411-431.

[14] E.Jerábek, Independent bases of admissible rules, Logic J. IGPL, 16(2008), 249-267. 
[15] R.Iemhoff, G.Metcalfe, Proof theory for admissible rules, Annals of Pure and Applied Logic, 159(2009), 171-186.

[16] R.Iemhoff, On the admissible rules of intuitionistic propositional logic, J. Symbolic Logic, 66(2001), 281-294.

[17] V.V.Rybakov, Projective formulas and unification in linear temporal logic LTLU, Logic J. $I G P L, \mathbf{2 2}(2014)$, no. 4, 665-672.

[18] S.Babenyshev, V. Rybakov, Unification in linear temporal logic LTL, Annals of Pure and Applied Logic, 162(2011), 991-1000.

[19] S.I.Bashmakov, A.V. Kosheleva, V. Rybakov, Projective formulas and unification in linear discrete temporal multi-agent logics, Sib. Electronic Math. Reports, 13(2016), 923-929.

[20] F.Baader, S.Ghilardi, Unification in modal and description logics, Logic J. IGPL, 19(2011), $705-730$.

[21] F. Baader, W. Snyder, Unification theory, in: A. Robinson, A. Voronkov (Eds.), Handbook of Automated Reasoning, vol. I, Elsevier, 2001, 445-533.

[22] W.Dzik, Unitary Unification of S5 Modal Logic and its Extensions, Bull. Section of Logic, 32(2003), no. 1-2, 19-26.

[23] E.Jerábek, Blending margins: the modal logic K has nullary unification type, J. Logic Comput., 25(2015), 1231-1240.

[24] F.Wolter, M.Zakharyaschev, Undecidability of the unification and admissibility problems for modal and description logics, ACM Transactions on Comput. Logic, 9(2008), no. 4, article no. 25 .

[25] W.A.Pogorzelski, P.Wojtylak, Completeness theory for propositional logics, Birkhäuser Basel, 2008.

[26] S.I.Bashmakov, A.V.Kosheleva, V.Rybakov, Unification for multi-agent temporal logics with universal modality, J. of Logics and their Application, 4(2017), no. 4, 939-954.

\section{Унификация в линейной модальной логике нетранзитивного времени с универсальной модальностью}

Степан И. Башмаков

Институт математики и фундаментальной информатики Сибирский федеральный университет Свободный, 79, Красноярск, 660041

Россия

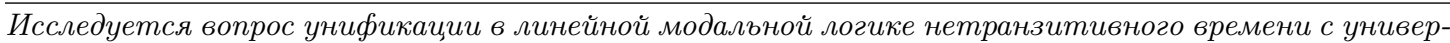
сальной модальностъю. Предлагается семантическое построение логики на линейных нетранзитивных фреймах Крипке, эффективная определимость и проективность унифицируемых формул. Найден алгоритм построения наиболее общего унификатора.

Ключевые слова: временная модальная логика, нетранзитивный фрейм Крипке, унификаиия, граунд-унификатор, проективные формулы. 